\title{
CARACTERIZACIÓN ESPACIO TEMPORAL DEL CAMPO DE VIENTOS SUPERFICIALES DEL PACÍFICO COLOMBIANO Y EL GOLFO DE PANAMÁ A PARTIR DE SENSORES REMOTOS Y DATOS IN SITU
}

\section{SPACE-TIME CHARACTERIZATION OF THE SURFACE WND FIELDS OVER THE COLOMBIAN PACIFIC AND GULF OF PANAMA USING REMOTE SENSING AND IN STU DATA}

\author{
J uan Gabriel Rueda Bayona1,Efraín Rodríguez-Rubio²,Javier Roberto Ortiz Galvis ${ }^{3}$ \\ Área de Oceanografía Operacional, \\ Dirección General Marítima - Centro Control Contaminación del Pacífico (DIMAR-CCCP), \\ Vía El Morro, Capitanía de Puerto de Tumaco, San Andrés de Tumaco, Nariño, Colombia. \\ juan_rueda_mof@hotmail.com ${ }^{1}$, erodiguezrubio@yahoo.com.ar ${ }^{2}$,j.Ortiz@dimar.mil.co ${ }^{3}$
}

Rueda, J., E. Rodríguez-Rubio y J. Ortiz. 2007. Caracterización espacio temporal del campo de vientos superficiales del Pacífico colombiano y el golfo de Panamá a partir de sensores remotos y datos in situ. Boletín Científico CCCP, (14): 49-68

Recibido en mayo de 2006; aceptado en diciembre de 2007

\section{RESUMEN}

La cuenca Pacífica Colombiana (CPC) se caracteriza por la influencia de los vientos Alisios del Norte y del Sur, los cuales generan la denominada Zona de Convergencia Intertropical (ZCIT). Esta dinámica, presente en los vectores de viento en la región, está asociada con fuerte actividad convectiva atmosférica, que explica los altos índices de pluviosidad de la región. Además, se considera de gran importancia identificar el comportamiento de los vientos superficiales en el golfo de Panamá, teniendo en cuenta que en esta región se desarrollan procesos atmosféricos de importante magnitud, como lo es el chorro de viento de Panamá. El presente trabajo cuantifica el campo de vientos horizontales de la zona marítima y costera del Pacífico colombiano mediante información in situ e información satelital, a una escala espacial de $25 \mathrm{~km}$. Además, se obtuvo el comportamiento anual, semestral y multianual de las componentes zonal y meridional del viento horizontal en superficie sobre las áreas de estudio. Se efectuó un análisis de correlación entre la información in situ y de satélite de marzo de 2006. La climatología establecida indica que la CPC y el golfo de Panamá presentan un comportamiento estacional semestral. Durante el primer semestre, predominan los vientos Alisios del Noroeste, con intensidades promedio entre los 5 y $7 \mathrm{~m}^{-1} \mathrm{~s}^{-1}$; durante este período se manifiesta el chorro de Panamá sobre la CPC, regulando sus condiciones oceanográficas. En el segundo semestre, empezando en julio y hasta diciembre, la ZCIT se ausenta de la CPC, al igual que el chorro de Panamá, presentándose predominio de vientos del suroeste del chorro del Chocó sobre las áreas de estudio. Finalmente, se presenta la relación entre las magnitudes anuales y semestrales de la CPC y el chorro del Chocó.

PALABRAS CLAVE: vientos Alisios, Zona de Convergencia Intertropical (ZCIT), cuenca Pacífica Colombiana (CPC), golfo de Panamá, chorro de viento de Panamá, chorro de viento del Chocó.

\footnotetext{
ABSTRACT

The Colombian Pacific Basin is characterized by the influence of the North and South Trade Winds, which generate the denominated Intertropical Convergence Zone. This presented dynamics in the wind vectors on the region, is associated with a strong convective activity, explaining the high pluviosity indexes over that region. Also, it is considered of great importance to identify the behaviour of the surface winds in the Gulf of Panama, keeping in mind that in this region atmospheric processes of important magnitude are made, as it is it the Panama wind jet. The present work quantifies the horizontal winds field of the Colombian Pacific marine and coastal zone by means of in situ and satellite data, with a $25 \mathrm{Km}$ of spatial scale. In addition, it is obtained the annual, semiannual and multianual behavior of the zonal and southern superficial horizontal wind over the studied areas. It is developed a correlation analysis between the in situ and satellite data in march 2006. The established climatology indicates that Colombian Pacific Basin (CPB) and the Gulf of Panama show a semiannual seasonal behavior. During the first semester the North Trade winds predominate, with average intensities between 5 and $7 \mathrm{~m} . \mathrm{s}^{-1}$, during this period the Panama wind jet is detected on the CPB, regulating the CPB oceanographic conditions. In the second semester (July-December), the ITCZ and the Panama Wind jet remain absented on the CPB, being presented prevalence of South-west Trade winds of the Choco wind jet on the study areas. Finally it is present the relation between the annual and semiannual magnitudes of the CPB and the Chocó wind jet.

KEY WORDS: Trade Winds, Intertropical Convergence Zone (ITCZ), Colombian Pacific Basin (CPB), Gulf of Panama, Panama wind jet, Chocó wind jet.
} 


\section{INTRODUCCIÓN}

La CPC, delimitada por las latitudes $1^{\circ} 30^{\prime} \mathrm{N}$ a $7^{\circ} 10^{\prime} \mathrm{N}$ y longitudes $77^{\circ} 40^{\prime} \mathrm{W}$ y $84^{\circ} \mathrm{W}$ (Fig. 1 ), se caracteriza por la presencia de vientos Alisios del Norte al principio y al final del año, y de vientos Alisios del Sudoeste durante el resto del año, mostrando el desplazamiento de la ZCIT, la cual se desplaza hacia el Sur durante el primer trimestre del año y hacia el Norte durante el segundo semestre del mismo. Este desplazamiento de la ZCIT está asociado con una fuerte y frecuente pluviosidad, debido a la alta actividad de convección en la atmósfera y a la condensación resultante (Forsbergh, 1969; Eslava, 1994), regulando el comportamiento de la interacción océano-atmósfera de la región (Umatani y Yamagata 1991; Waliser y Somerville, 1994; Poveda y Mesa, 1997, 1999 y 2000; Rodríguez-Rubio et ál. 2003)

Esta dinámica física se encuentra bajo la influencia de eventos de macro escala espacial y temporal como el ENOS (El Niño - Oscilación del Sur), el cual altera, entre otros, el flujo regular de los vientos Alisios, los cuales modifican las condiciones océano-atmosféricas de la zona (Philander, 1990; Poveda 2004)

Es también de gran importancia, conocer la dinámica del campo de vientos en el golfo de Panamá, ya que en esta región se desarrollan procesos físicos como la dinámica del chorro de viento de Panamá, el cual afecta directamente el flujo de vientos meridional y zonal sobre la CPC (Rodríguez-Rubio y Stuardo, 2002; Rodríguez-Rubio et ál., 2003). Este chorro de viento es uno de los tres producidos por el paso del viento a través de depresiones en la cordillera Central en Tehuantepec (México), Papagayo (Nicaragua-Costa Rica) y Panamá (McCreary et ál., 1989; Chelton et ál., 2000a). Este chorro de viento es referido por Wang y Lee (2007) como Caribbean Low Level Jet (CLLJ)

Sólo hasta 1973, cuando aparecieron los sensores a bordo de satélites como el dispersómetro Seasat-A, se comenzaron a desarrollar estudios sobre campos de vientos. Estos sensores permiten la medición de la velocidad y dirección del viento con una precisión, cobertura y periodicidad nunca antes lograda para estudios de dinámica y variabilidad de las corrientes atmosféricas (Robinson, 1994; Topliss y Guymer, 1995). De esta manera se comenzó a profundizar en los estudios de los efectos de los chorros de vientos en la circulación oceánica, ya que estudios anteriores sólo contaban con la climatología mensual de vientos (Hellerman y Rosenstein, 1983) o modelos como el European Center for Medium-Range Weather Forecast (ECMWF) o como el Reanálisis de NCEP/NCAR, que al aplicarlos en modelos generales de circulación no resolvían bien los patrones de circulación en zonas influenciadas por estos chorros de vientos.

Las mediciones a través de dispersómetros de viento mejoran la resolución espacial y temporal, para efectos de estudiar el origen e impacto de los chorros de viento de Centroamérica en la circulación del océano Pacífico Tropical y la ensenada de Panamá (Chelton et ál., 2000a; Chelton et ál., 2000b; Rodríguez-Rubio y Stuardo, 2002). Por tanto esta tecnología satelital constituye una herramienta alternativa de gran precisión para resolver el campo de vientos en la CPC y el golfo de Panamá.

Los estudios de velocidad y dirección del viento, a partir de información in situ, para el océano Pacífico colombiano, se remontan a la expedición de la Comisión Interamericana del Atún Tropical y el Gobierno de Colombia, en el marco de la expedición denominada The Augmented Colombian El Niño Tuna Oceanography (ACENTO), durante 1963. 1965. En esta expedición se promediaron datos de viento en rejillas de $1^{\circ} \times 1^{\circ}$ con mucha dispersión de los datos originales en tiempo y espacio, limitando una estimación adecuada del campo de viento para la zona.

Desde 1972 la Dirección General Marítima (DIMAR) ha venido realizando cruceros oceanográficos en los cuales se han tomado datos de la magnitud y dirección del viento horizontal superficial en forma regular a partir de una rejilla relativamente uniforme, con separación aproximada de medio grado en latitud y longitud. Por lo anterior es oportuno efectuar un estudio del campo de vientos horizontales del Pacífico colombiano, que hasta ahora no se ha realizado.

Este trabajo pretende cuantificar la relación existente entre los datos in situ recolectados en las campañas oceanográficas y la información obtenida mediante tecnología satelital de avanzada, y tiene como principal objetivo estudiar las características espacio-temporales del campo de vientos en la CPC y el golfo de Panamá. Los resultados de esta investigación servirán de ayuda para la comprensión de la dinámica superficial de los campos de vientos 
horizontales de estas regiones, permitiendo establecer patrones físicos de comportamiento de los diferentes procesos oceanográficos y atmosféricos que se desarrollan en la región.

\section{ÁREA DE ESTUDIO}

El área de estudio comprende dos regiones, una es la CPC, determinada geográficamente entre los paralelos $1^{\circ} 30^{\prime}$ hasta los $7^{\circ} 10^{\prime}$ de latitud Norte y entre los $77^{\circ} 40^{\prime}$ a $84^{\circ} 00^{\prime} \mathrm{W}$, y en el golfo de Panamá se fija como zona de observación el área comprendida entre los $7^{\circ} \mathrm{N}$ a $9^{\circ} \mathrm{N}$ y las longitudes $78^{\circ} \mathrm{W}$ hasta los $81^{\circ} \mathrm{W}$.

La CPC limita al $N$ con las aguas del golfo de Panamá; al S con la dorsal de Carnegie y la costa de Ecuador; al E con la franja del litoral Pacífico colombiano (departamentos de Chocó, Valle del Cauca, Cauca y Nariño), y al $\mathrm{W}$ con las aguas ecuatoriales del océano Pacífico y la cordillera submarina de Los Cocos.

Las formaciones geomorfológicas más importantes de la CPC son las islas de Malpelo y Gorgona. La primera ubicada a $3^{\circ} 51^{\prime}$ de latitud N y $81^{\circ} 51^{\prime}$ 'de longitud W, a 270 millas náuticas al Oeste de Buenaventura. Esta isla es la única prolongación emergida del relieve oceánico submarino (la dorsal de Malpelo) que se eleva bruscamente desde fondos abisales. La isla Gorgona, ubicada sobre los $2^{\circ} 55^{\prime}$ y $3^{\circ} 00^{\prime}$ de latitud Norte y $78^{\circ} 09^{\prime}$ a $78^{\circ} 14^{\prime}$ ' de longitud Oeste, distante $30 \mathrm{~km}$ de la costa más cercana, está localizada a mitad de camino entre un océano de profundidades abisales de aguas transparentes y la contrastante zona costera, construida por planos aluviales que reciben cantidades considerables de agua dulce y sedimentos aportados por ríos caudalosos e innumerables quebradas (CCCP, 2002)

El litoral Pacífico colombiano (Fig. 1) ha sido sectorizado por regiones de la siguiente manera (CCCP, 2002):

Zona Norte: comprendida desde Punta Ardita hasta Pizarro (aproximadamente entre las latitudes $07^{\circ} 08^{\prime}$ y $\left.04^{\circ} 57^{\prime} \mathrm{N}\right)$. La región costera se denomina Zona Costera Norte (ZCN)

Zona Centro: comprendida desde Pizarro hasta Punta El Coco - Gorgona (aproximadamente entre latitudes $04^{\circ} 57^{\prime}$ y $03^{\circ} 00^{\prime} \mathrm{N}$ ). La región costera se denomina Zona Costera Centro (ZCC)

Zona Sur: comprendida desde Punta el Coco - Gorgona hasta Cabo Manglares (aproximadamente entre las latitudes $03^{\circ} 00^{\prime}$ y $01^{\circ} 36^{\prime} \mathrm{N}$ ). La región costera se denomina Zona Costera Sur (ZCS)

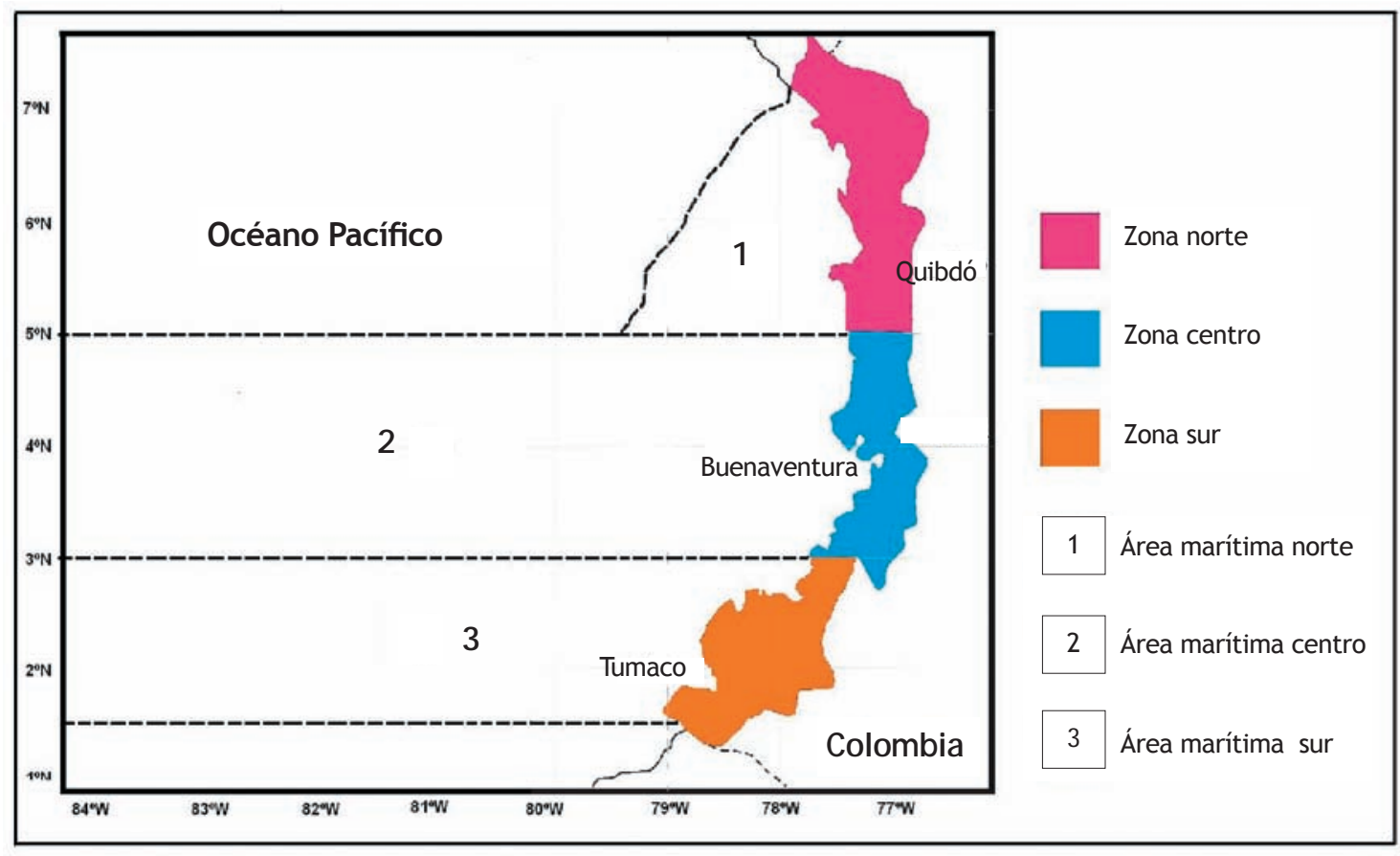

Figura 1. Delimitación de zonas en el Pacífico colombiano. 
Por su parte el golfo de Panamá, encerrado por el istmo, se delimita entre los $07^{\circ} 26^{\prime}$ a $9^{\circ}$ de latitud $\mathrm{N}$ y los $78^{\circ} 10^{\prime}$ a $80^{\circ} 29^{\prime}$ de longitud W (Smayda, 1966). Esta región se encuentra bajo la influencia de los vientos Alisios de los hemisferios Norte y Sur (Kwiecinski et ál, 1975; Tokioka, 1983). El conocimiento de la dinámica del campo de vientos es de gran importancia, debido que esta región se encuentra conectada a la dinámica océanoatmosférica de la CPC (Chelton et ál., 2000a)

\section{METODOLOGÍA}

Para la obtención de los datos in situ a partir de cruceros oceanográficos, se estableció una rejilla o puntos de muestreo en la CPC, donde cada punto corresponde a una estación (Fig. 2)

La recolección de la información de vientos empezó a partir de 1972, con una rejilla de 114 estaciones. Posteriormente, estos puntos de muestreo se redujeron a 44, ya que estudios realizados por el Centro Control Contaminación del Pacífico (CCCP) permitieron determinar zonas homogéneas dentro de la CPC, agrupando estaciones con información oceanográfica similar (Villegas, 2003)

\section{Obtención de datos satelitales}

El dispersómetro de microondas QuikScat (QSCAT) fue lanzado en junio de 1999, a bordo del satélite QuickBird, con el objetivo de medir

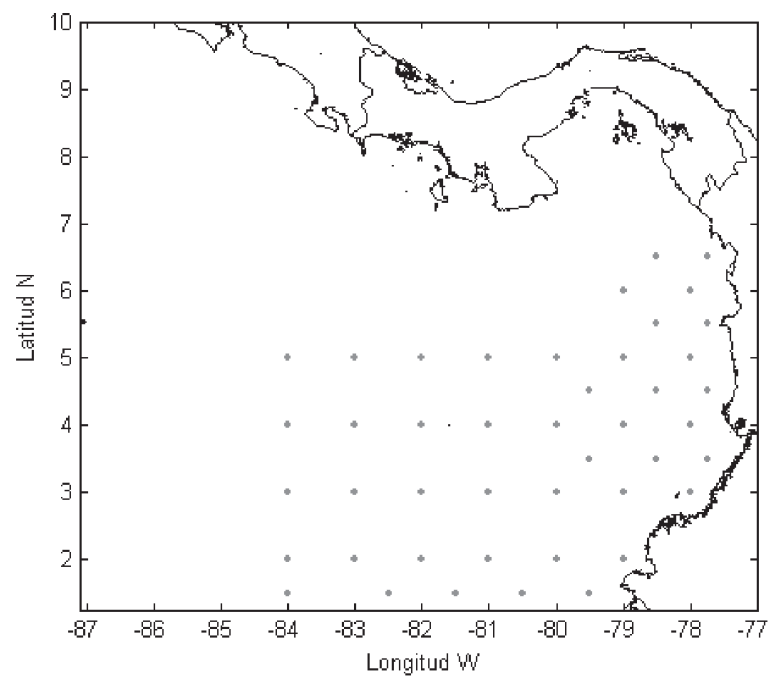

Figura 2. Estaciones de muestreo en la CPC. los vientos superficiales del océano. Los sensores remotos de SeaWinds están constituidos básicamente por radares que transmiten pulsos de microonda hacia la superficie de la Tierra y miden la energía que regresa dispersada. La resolución de las imágenes satelitales es de $0.25^{\circ}$ latitud por $0.25^{\circ}$ longitud.

La base de datos QSCAT comenzó a ser construida desde junio de 1999, siendo actualizada diariamente. La información utilizada en este estudio fue suministrada por la empresa de investigación Remote Sensing Systems, la cual almacena los datos de viento de todo el globo terráqueo en un solo archivo binario que para efectos del presente trabajo se debió recortar de acuerdo al área de estudio definida en esta investigación.

Una vez determinada el área de estudio y obtenida la información, se inició por aplicar la convención oceanográfica para la interpretación y orientación de los componentes zonal (u) y meridional (v) del vector de vientos horizontales. Para ello se usaron las siguientes expresiones:

$$
\begin{aligned}
& u=m^{*} \cos (90-d) * \pi / 180 \\
& v=m^{*} \operatorname{sen}(90-d) * \pi / 180 \\
& \text { donde, } \\
& m \text { es la magnitud. } \\
& d \text { representa la dirección. }
\end{aligned}
$$

La información de la temperatura superficial del mar (TSM) del promedio mensual de marzo de 2006 fue descargada vía internet del sitio de la empresa Remote Sensing Systems, cuyos datos provienen del satélite Tropical Rainfall Measuring Mision (TRMM), a bordo del cual se encuentra el sensor TRMM Microwave Imager (TMI), un radiómetro de microondas pasivo, cuyas imágenes tienen una resolución espacial de $0.25^{\circ}$ por píxel $(25 \mathrm{~km})$

Tanto los datos de vientos como la temperatura superficial del mar fueron procesados y analizados en el programa matemático MATLAB.

\section{Obtención de datos in situ}

La información in situ fue obtenida por medio de medidores meteorológicos manuales y automáticos. Los medidores manuales son el anemómetro y la veleta. Para medir la dirección y velocidad del viento se tuvo en cuenta el viento real y el viento aparente 
dentro del buque oceanográfico, con el propósito de evitar registro espurio.

Los sensores meteorológicos automáticos fueron instalados en la parte más alta del buque, con el objeto de evitar interferencias en la obtención del registro. Al momento de tomar el dato de viento se hicieron simultáneamente registros con las herramientas manuales y automáticas. Se compararon los resultados para así almacenar en la base de datos el resultado obtenido por el sensor.

Mediante la metodología de Middleton (2000) se implementaron ecuaciones en MATLAB, para la representación del comportamiento de los vectores de viento.

La dirección promedio $(\mathrm{T})$ para los datos in situ fue obtenida mediante la expresión matemática:

$$
T=\operatorname{arcTan}(v / u)
$$

donde,

$u$ es la componente zonal.

$v$ la componente meridional de los vectores de viento horizontal.

El cálculo de la persistencia del viento se obtuvo por medio de la expresión:

$\mathrm{L}=100 * R / N$

donde,

$L$ es la persistencia.

$\mathrm{N}$ es el número de observaciomes

$R$ es la magnitud del vector medio, expresado como:

$R=\sqrt{u^{2}+v^{2}}$
Los resultados del cálculo de probabilidad del viento, en los cuales si $\mathrm{p}<0$, el valor arrojado se considera un valor nulo, debido a que estos deben estar entre un rango de 0 a 1 . La ecuación utilizada para determinar la probabilidad es:

$$
\mathrm{p}=1-\exp \left(-R^{*} R / N\right)
$$

En cada imagen obtenida del campo de vientos de la CPC, a partir de información in situ, abril y julio fueron los de menor cubrimiento con respecto a los 44 puntos de muestreo. Septiembre es el único mes que hasta el 2007 posee el cubrimiento total de las 44 estaciones de la rejilla de muestreo sobre la CPC (Fig. 3)

En el filtro aplicado a los datos in situ se consideró que un dato vectorial debería tener una orientación entre los $0^{\circ}$ a $90^{\circ}$, en cualquiera de los cuadrantes de orientación geográfica, con respecto a la tendencia direccional de los vectores más cercanos. Es decir, el vector no podrá exceder el límite de los rangos en el componente meridional y zonal promedio del grupo de datos de dirección, aunque se hace la excepción de esta regla en las áreas de observación que estén bajo la influencia de la ZCIT o que puedan estar cercanas a la zona costera marítima.

Para el análisis del campo de viento de marzo de 2006 se utilizó el programa Surfer, en el cual se utilizó el método de interpolación vecino natural o natural neighbour, expresado por medio de la ecuación:

$G(x, y)=\sum_{i=1}^{n} w_{i} f\left(x_{i}, y_{i}\right)$

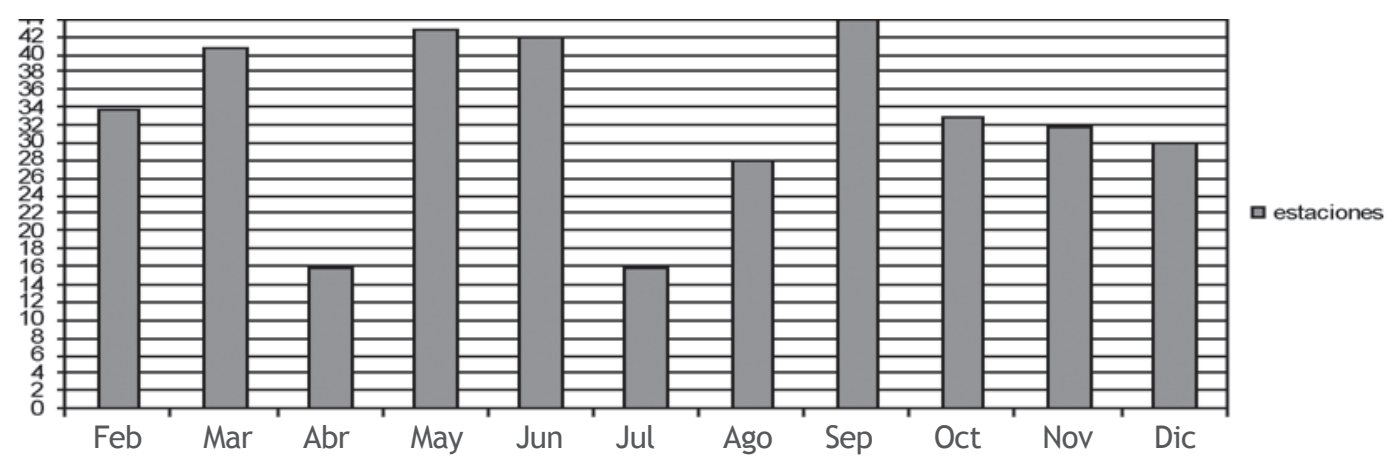

Figura 3. Cubrimiento máximo de estaciones en cada gráfica de campo de vientos 1972-2007. 
donde,

$G(x, y)$ es la estimación del vecino natural en $(x, y)$

N es el número de vecinos más cercanos (nearest neigbours) usados para la interpolación.

$f\left(x_{i}, y_{i}\right)$ es el valor observado en $\left(x_{i}, y_{i}\right)$

$W_{i}$ es el peso asociado con $f\left(x_{i}, y_{i}\right)$, calculado a partir de la ecuación:

wi $(x)=\frac{\operatorname{Vol}\left(V_{p i} \cap V_{x}^{+}\right)}{\operatorname{Vol}\left(V_{x}^{+}\right)}$

donde,

Vol $\left(\mathrm{V}_{\mathrm{pi}}\right)$ representa al volumen de la celda de Voronoi $\mathrm{V}_{\mathrm{pi}}$

$\mathrm{V}_{\mathrm{x}}{ }^{+}$es la celda de Voronoi modificada por la

inserción un punto en $(x, y)$

Para cualquier $\left(\mathrm{x}_{\mathrm{i}}, \mathrm{y}_{\mathrm{i}}\right)$ el valor del peso asociado wi $\left(\mathrm{x}_{\mathrm{i}}, \mathrm{y}_{\mathrm{i}}\right)$ siempre estará entre 0 y 1 ; valor de 0 cuando el punto de inserción $\mathrm{pi}=\left(\mathrm{x}_{\mathrm{i}}, \mathrm{y}_{\mathrm{i}}\right)$ no es un vecino natural de $(x, y)$, y 1 cuando pi está exactamente ubicado en la misma posición de $(x, y)$ (Ledoux y Gold, 2004)

Para conocer el grado de similitud entre los datos de viento satelitales y los obtenidos en los cruceros oceanográficos, se procedió a realizar correlaciones de las direcciones (azimut) entre ambas fuentes de datos. Debido a que el análisis de correlación de la estadística estándar no es aplicable a datos de tipo direccional (Jones, 2006), se procedió a utilizar la correlación circular en donde se considera la asociación entre dos variables circulares aleatorias, cuya asociación se puede establecer mediante el método Embedding de correlación canónica propuesto por Mardia y Jupp (2000). Este método fue implementado por Jones (2006) en rutinas bajo el entorno de programación MATLAB, las cuales fueron utilizadas en este estudio.

\section{RESULTADOS}

Se procesó la información del sensor remoto QSCAT del período 1999-2007, para obtener información de los vectores de viento en la CPC con el objeto de correlacionar con los datos in situ del CCCP 1972-2007. Esta relación permitió comparar el campo direccional de la información de vientos obtenida durante los cruceros oceanográficos con respecto a los obtenidos por el sensor remoto, presentándose una buena correspondencia entre ambas fuentes de datos de viento para los meses en donde se pudieron obtener datos coincidentes, tanto en espacio y en tiempo, lo cual se aprecia en los resultados de la correlación canónica consignados en la Tabla I.

Los resultados de dicha tabla muestran una buena correlación de la dirección de los vientos en gran parte del año; tan sólo los meses de abril, mayo, noviembre y diciembre presentaron baja correlación y no alcanzaron a rechazar la hipótesis de independencia en los datos.

Tabla I. Resultado de la correlación canónica entre direcciones del viento QSCAT e in situ obtenidas a partir de la rejilla de muestreo.

\begin{tabular}{ccccc}
\hline Meses & $\mathbf{R}^{2}$ & $\begin{array}{c}\text { Signifi- } \\
\text { cancia: } \\
\text { Alfa }\end{array}$ & $\begin{array}{c}\text { Ho: datos } \\
\text { son indepen- } \\
\text { dientes }\end{array}$ & $\begin{array}{c}\text { Pares de } \\
\text { esta- } \\
\text { ciones: N }\end{array}$ \\
\hline Feb. & 0.583 & 0.05 & Rechaza & 42 \\
Mar. & 0.559 & 0.05 & Rechaza & 43 \\
Abr. & 0.121 & 0.05 & Acepta & 16 \\
May. & 0.107 & 0.05 & Acepta & 43 \\
Jun. & 0.297 & 0.05 & Rechaza & 38 \\
Jul. & 0.663 & 0.05 & Rechaza & 16 \\
Ago. & 0.694 & 0.05 & Rechaza & 28 \\
Sep. & 0.871 & 0.05 & Rechaza & 44 \\
Oct. & 0.401 & 0.05 & Rechaza & 25 \\
Nov. & 0.21 & 0.05 & Acepta & 32 \\
Dic. & 0.167 & 0.05 & Acepta & 30 \\
\hline
\end{tabular}

Ho: Hipótesis nula

Se obvió la información de magnitud en las imágenes de datos in situ, debido a que los registros de datos de viento eran muy puntuales (dato de hora por día), generando incertidumbre en la información de la magnitud, la cual se ve supeditada a fenómenos de micro-escala como las ráfagas de viento, a diferencia de la dirección de los vectores, la cual es mucho más regular. 


\section{1) Comportamiento del campo de vientos del golfo de Panamá y su incidencia sobre la ZCIT en la CPC}

Con respecto al campo de vientos del golfo de Panamá se encontró un comportamiento estacional semestral. Durante el primer semestre predominaron los vientos Alisios del noroeste, con intensidades promedio entre los 5 y $7 \mathrm{~m}^{-1}{ }^{-1}$ (Fig. 4). Durante este semestre se manifiesta el chorro de Panamá sobre la CPC, regulando el desplazamiento de la ZCIT sobre el golfo de Panamá y la CPC, incidiendo además sobre las componentes zonales y meridionales de la región. Estos resultados encuentran relación con los estudios realizados por Rodríguez-Rubio y Stuardo (2002); Rodríguez-Rubio et ál. (2003); Garcés-Vargas et ál. (2004), los cuales indican la manifestación de la ZCIT sobre las áreas anteriormente mencionadas.

Durante los dos primeros meses del segundo semestre (Jul. - Ago.) los vectores de vientos superficiales, predominantes en su mayoría del noroeste durante el primer semestre, disminuyeron significativamente su componente meridional Norte (Fig. 5). Esta disminución del componente meridional Norte permite, a partir de septiembre y hasta octubre, la incursión de vientos Alisios del suroeste en el golfo de Panamá. A partir de noviembre se dio la reactivación del chorro de viento de Panamá, manifestándose sobre el golfo panameño, hasta julio con vientos predominantes del noroeste (Fig. 5)

Por lo anterior, se nota claramente que el chorro de viento de Panamá inicia su desarrollo a partir de noviembre, manifestando su mayor intensidad sobre el golfo en enero, para posteriormente disminuir de manera gradual desde febrero hasta junio (Fig. 5)

Esta incidencia del chorro de Panamá sobre el golfo panameño, denotado como el flujo constante de los vientos Alisios del Norte a través del istmo, transporta agua superficial fuera del golfo, generando disminución en la temperatura superficial del mar (TSM), la cual se aprecia en la imagen de TSM de marzo de 2006, permitiendo establecer un comportamiento similar en la TSM a los estudios realizados por Kwiecinski et ál., (1975) y Legekis (1985)

Con relación al comportamiento de la ZCIT en la CPC (Fig. 5), durante enero ésta actuó sobre la ZCC. Los vientos predominantes en la CPC durante este mes fueron del noroeste, Oeste y suroeste, con intensidades promedio entre los $3 \mathrm{y}$ $6 \mathrm{~m} . \mathrm{s}^{-1}$. En febrero la ZCIT llega hasta la ZCS, con intensidades entre los 3 y $4 \mathrm{~m} \cdot \mathrm{s}^{-1}$, presentando vientos predominantes del noroeste. En marzo la ZCIT regresa nuevamente a la ZCC, pero con menores intensidades de viento a las registradas durante los dos primeros meses del año.
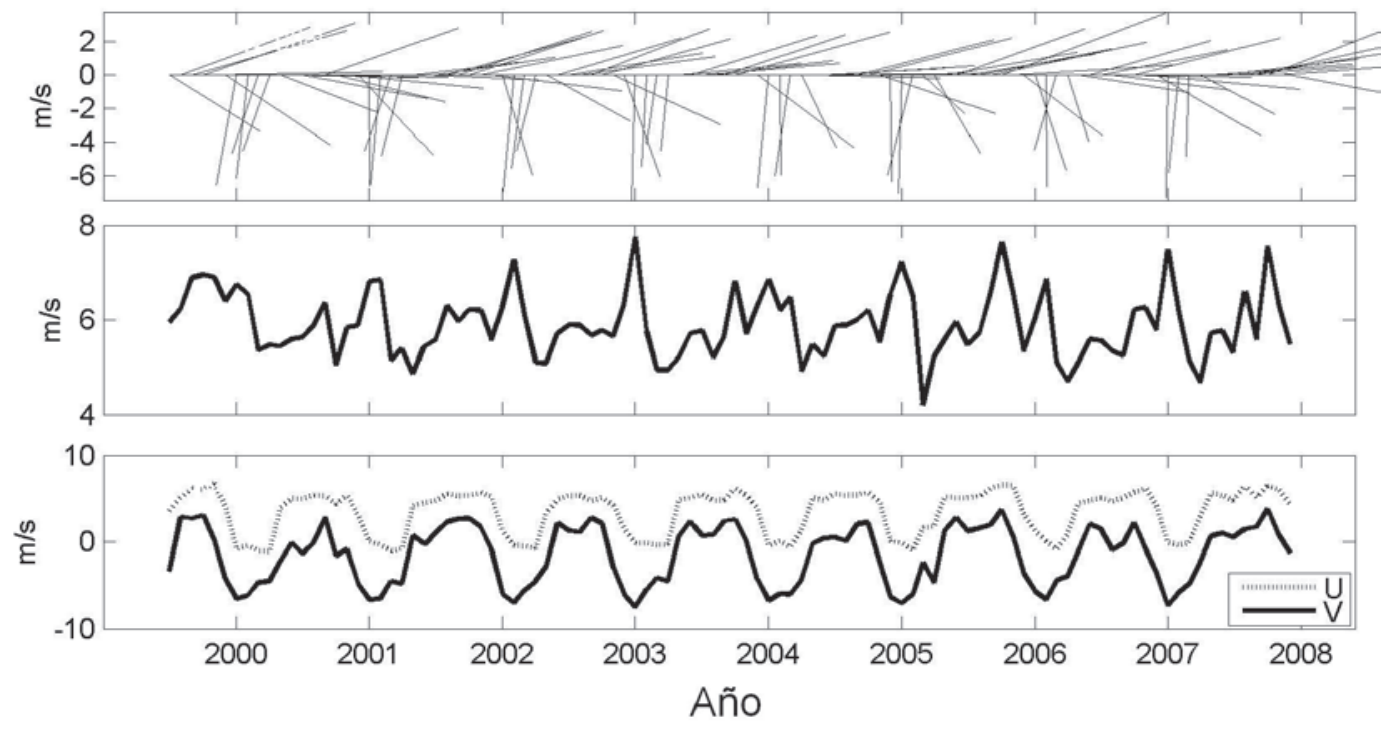

Figura 4. Comportamiento multianual de los vectores de viento del golfo de Panamá (a) Dirección,(b) Magnitud. 

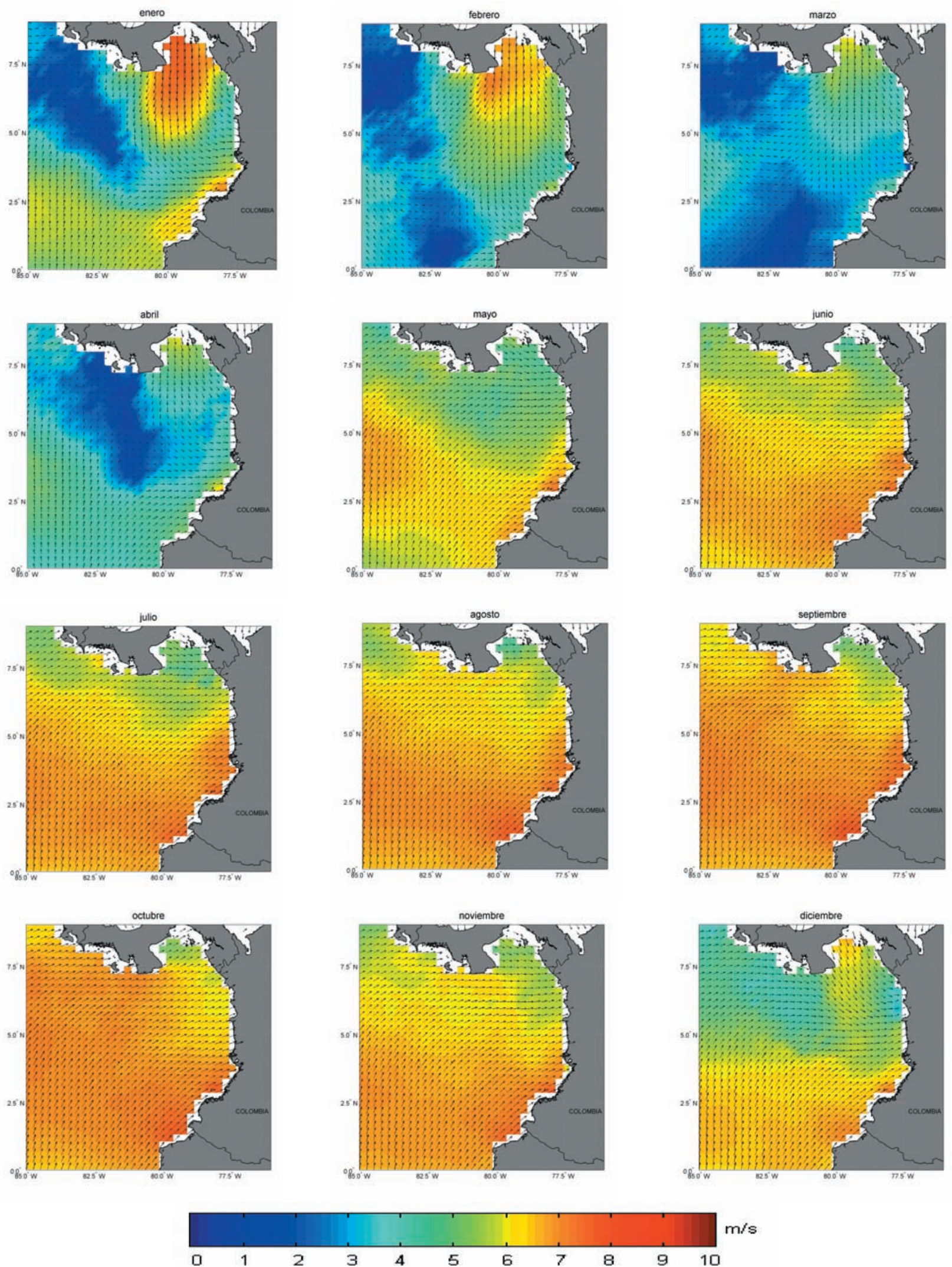

Figura 5. Serie de tiempo de datos satelitales QSCAT para el período de 101 meses (Jul. 1999 a Nov. 2007) del campo de vientos promedio mensual en la CPC y el golfo de Panamá. 
Entre marzo y junio la ZCIT se manifiestó sobre la CPC, ascendiendo latitudinalmente, llegando hasta el golfo de Panamá en julio. Finalmente, a partir de agosto y hasta noviembre, la ZCIT dejó de manifestarse sobre la CPC, al igual que el chorro de Panamá. El anterior comportamiento se puede relacionar con los estudios de campo de vientos realizados en las costas de América Central por Chelton et ál. 2000a, los cuales indican la influencia del chorro de Panamá sobre el área de estudio.

A diferencia del estudio del campo de vientos realizado por Malikov (1998), mediante información con resolución espacial de $2.5^{\circ}$ originarios de la NOAA y su respectiva caracterización mediante un modelo numérico del Centro Meteorológico de Europa, la ZCIT no se comportó como se presenta en los resultados de dicho trabajo; manifestándose como un cinturón irregular de bajas velocidades sobre la CPC, identificado a partir de la información de QSCAT (Fig. 5)

\section{2) Comportamiento mensual del campo de vientos de la CPC}

\section{Campo de vientos mensual en la CPC}

\section{Enero}

El campo de vientos obtenido para enero (Fig. 5) presentó la directa influencia del chorro de Panamá sobre la CPC, registrándose velocidades en los vectores de viento entre los 6 y $9 \mathrm{~m} . \mathrm{s}^{-1}$.

Con respecto a la ZCN del Pacífico colombiano (Fig.1) se apreciaron direcciones de viento provenientes del noroeste, con velocidades entre los 4 y $6 \mathrm{~m} \cdot \mathrm{s}^{-1}$. La ZCC registró vientos predominantes del oeste, presentando magnitudes en su gran mayoría de $5 \mathrm{~m} . \mathrm{s}^{-1}$. Con relación al comportamiento de los vientos en el Sur del litoral Pacífico colombiano, los vectores predominantes fueron del suroeste, incrementando sus velocidades a medida que disminuye la latitud. La región oceánica de la CPC se vio directamente influenciada por el efecto del chorro de Panamá, presentándose velocidades entre los $6 \mathrm{a} 9 \mathrm{~m} . \mathrm{s}^{-1}$ en la región oceánica norte.

La ZCIT se manifestó atravesando la CPC por medio de un cinturón con bajas velocidades entre 0 y $4 \mathrm{~m} \cdot \mathrm{s}^{-1}$. En las regiones en donde el viento superó los $3 \mathrm{~m} . \mathrm{s}^{-1}$ los vectores provenían del noroeste; los vientos inferiores a $3 \mathrm{~m} \cdot \mathrm{s}^{-1}$ no tienen una orientación definida, por lo cual se les puede denominar como vientos en calma a ligeros.

\section{Febrero}

En febrero (Fig. 5) el comportamiento obtenido de los vectores de viento mantuvo la influencia del chorro de Panamá sobre la CPC, en donde las intensidades disminuyeron ligeramente en el golfo de Panamá. La CPC registró el ingreso de la ZCIT entre los meridianos $81^{\circ}$ a $84^{\circ} \mathrm{W}$, presentándose disminución en las intensidades del viento con valores de 0 a $4 \mathrm{~m} \cdot \mathrm{s}^{-1}$. En la región oceánica Sur de la CPC se presentó un núcleo de bajas intensidades, por lo que se puede considerar como una zona con vientos en calma a brisa moderada, acuerdo escala Beaufort.

Para febrero la ZCN (Fig. 5) registró vientos con

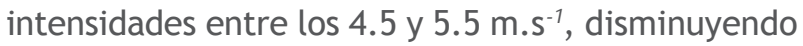
levemente con respecto al mes anterior. Los vectores predominantes provenían del nor-noroeste. La ZCC presentó un comportamiento similar con respecto a la ZCN, aunque ésta registró menores intensidades en sus velocidades, entre los 3 a $5 \mathrm{~m} . \mathrm{s}^{-1}$; por último se presentaron en esta zona vientos procedentes del noroeste a medida que disminuye la latitud.

La ZCS disminuyó las intensidades registradas de 7 a $8 \mathrm{~m} . \mathrm{s}^{-1}$ para enero, hasta $4.5 \mathrm{a} 6 \mathrm{~m} \cdot \mathrm{s}^{-1}$ para febrero. La orientación de los vectores cambió notoriamente con predominio del noroeste, a diferencia de enero cuando hubo predominio del suroeste.

La información in situ para este mes registró, igualmente, la influencia del chorro de Panamá y la manifestación de la ZCIT sobre la CPC. Se registraron vientos predominantes del nor-noreste en la ZCN y ZCC; la región sur del área de estudio presentó irregularidad en dirección de los vectores de viento (Fig. 6), debido principalmente a la convergencia generada por los vientos Alisios del norte y sur en el área, generando zonas de alta actividad convectiva atmosférica (figuras 5 y 6 )

\section{Marzo}

El campo de vientos de marzo presentó la persistencia de vientos provenientes del noroeste para la ZCN, con vientos predominantes del Norte y nor-noroeste, con velocidades en la CPC entre 1 y $4 \mathrm{~m} \cdot \mathrm{s}^{-1}$ (Fig. 5)

La ZCC manifestó la influencia directa de la ZCIT, registrando vientos del noroeste y Oeste en gran parte de la región. En el área oceánica centro, 
tanto en los datos QSCAT y CCCP, el comportamiento de los vientos fue similar, registrándose entre los meridianos $79^{\circ}$ y $84^{\circ} \mathrm{W}$ vientos procedentes del noreste. La ZCS presentó el predominio de vientos del noroeste y a medida que la latitud disminuye, incursionaron vientos procedentes del oestenoroeste a las áreas cercanas a la costa. En la región oceánica Sur se registraron, de Norte a sur, vientos del noroeste, norte, noreste y en mayor proporción vientos del este.

Con relación a los resultados obtenidos mediante la información in situ, marzo registró el predominio de vientos del noreste en la región central de la CPC. La región costera presentó irregularidad en la dirección del viento. Esto se debe principalmente a la periodicidad en los muestreos, la cual se considera irregular por la baja frecuencia en el registro para identificar un comportamiento definido del patrón del viento, como también a los procesos océanoatmosféricos de micro-escala como son las brisas de mar y de tierra; los cuales dependiendo de la radiación solar del área costera y los sistemas de presión atmosférica local regulan el flujo de las masas de aire en la zona, afectando el patrón de viento del lugar, independientemente del comportamiento de los vectores de viento a meso-escala.

\section{Abril}

El comportamiento de los vectores de viento para abril (Fig. 5) presentó la influencia de la ZCIT sobre el litoral en la ZCN y la ZCC. Se identificaron vientos cercanos a la costa entre los 3.5 y $4.5 \mathrm{~m} . \mathrm{s}^{-1}$; las velocidades disminuyen a medida que aumenta la latitud en esta región. En la zona oceánica los vectores de vientos presentan velocidades un poco más altas, orientados norte-sur. La ZCC registró en el litoral vientos predominantes del Oeste como en ciertas áreas de su región oceánica. La ZCS presentó vientos con velocidades entre los 4 y $6 \mathrm{~m} . \mathrm{s}^{-1}$, procedentes del sureste, y a mayores latitudes predominantes del este. La ZCIT para abril atravesó toda la CPC, actuando de manera directa sobre las zonas costeras mencionadas, y a la vez ascendiendo latitudinalmente de manera gradual la cuenca colombiana.

Los vectores de viento obtenidos mediante información in situ (Fig. 6) presentaron en abril vientos predominantes del Sur en varias de las estaciones de muestreo. El comportamiento de los registros de dirección evidenció la zona de influencia de la ZCIT sobre la CPC. Aunque no es claro apreciar el área de convectividad en la información in situ, el campo de vientos obtenido mediante el sensor para este mes (Fig. 5) indicó claramente la ZCIT sobre la CPC, en donde se observa un debilitamiento en la magnitud del viento en la mayoría del área de estudio y la convección de los vectores de viento en la ZCC y ZCS de la CPC.

\section{Mayo}

Vientos al Norte de la CPC registran magnitudes entre los 4 y $5.5 \mathrm{~m} . \mathrm{s}^{-1}$, predominantes del Oeste y suroeste. En la ZCC las magnitudes de los vectores de viento aumentaron considerablemente cerca al litoral, en la latitud $3^{\circ} \mathrm{N}$, los cuales registraron velocidades entre los $6.5 \mathrm{a} 8 \mathrm{~m} . \mathrm{s}^{-1}$. El comportamiento registrado en la ZCS presentó también incrementos en la intensidad del viento con respecto al mes anterior, con velocidades que varían entre los 6 y $7 \mathrm{~m} . \mathrm{s}^{-1}$. De modo general, el mayor incremento se dio en las regiones centro y Sur de la CPC, lo cual indica el debilitamiento del chorro de Panamá, ascenso de la ZCIT y la intensificación de los vientos Alisios del Sur en el chorro del Chocó (Poveda y Mesa, 1999)

La información in situ obtenida para mayo (Fig. 6) indica que en la mayor parte de la CPC los vectores de viento son predominantes del Sur y suroeste (Fig. 5)

\section{J unio}

En junio las intensidades en los vectores de viento aumentaron gradualmente. La componente zonal se hizo más intensa, como a su vez disminuyó el componente meridional en la región del golfo de Panamá (Fig. 5). Esto indica la ausencia de la influencia del chorro de Panamá sobre la CPC y la incursión de los vientos Alisios del Sur a cargo del chorro del Chocó.

El comportamiento vectorial del campo de vientos en las regiones costeras fue muy similar, excepto en la región oceánica Sur de la CPC, en donde se presentan vientos predominantes del sur-suroeste. Las magnitudes de los vientos en la CPC exhiben valores máximos entre $8 \mathrm{a} 9 \mathrm{~m} . \mathrm{s}^{-1}$ en la ZCS y los más bajos en la ZCN con valores entre los 5 y $6 \mathrm{~m} . \mathrm{s}^{-1}$.

Los resultados obtenidos del campo de vientos mediante información in situ evidenciaron vientos del sureste, Sur y suroeste en las regiones oceánicas de la CPC, lo que se materializó con la manifestación del chorro del Chocó sobre la CPC (Fig. 6) 


\section{J ulio}

El comportamiento de los vectores de viento para julio presentaron en la ZCN (Fig. 5) vientos con intensidades entre los 5.5 y $7.5 \mathrm{~m} . \mathrm{s}^{-1}$, con dirección oeste-suroeste. La ZCC manifiestó mayores intensidades, con vientos de 7 a $9 \mathrm{~m} . \mathrm{s}^{-1}$. Este incremento se aprecia a medida que disminuye la latitud en esta región costera. La ZCS presentó un comportamiento similar a la zona centro, aunque con mayores intensidades y mayor procedencia del sur. De manera general, julio manifestó un campo de vientos muy similar al del mes anterior, con vectores de viento muy similares.

El campo de viento de julio a partir de información in situ (Fig. 6) registró un comportamiento vectorial similar al obtenido mediante el sensor satelital (Fig. 5). Predominaron los vientos Alisios del suroeste en la mayoría de estaciones muestreadas, los cuales incrementaron su componente zonal Oeste disminuyendo su componente meridional sur, a medida que aumentó la latitud sobre el área de estudio.

\section{Agosto}

En agosto los vientos Alisios del Sur siguieron incrementando gradualmente sus intensidades, manteniendo por toda la CPC vectores procedentes del suroeste (Fig. 5)

La ZCN presentó vientos con intensidades entre los 6 y $7 \mathrm{~m} \cdot \mathrm{s}^{-1}$, a diferencia de su región oceánica, registrando velocidades menores. La ZCC presentó intensidades mayores que la región norte, con velocidades entre los 6.5 y $8 \mathrm{~m} . \mathrm{s}^{-1}$; la región oceánica centro tuvo un comportamiento similar al de la región oceánica norte. La ZCS presentó intensidades un poco mayores que la ZCC, oscilando entre los 7 y $8 \mathrm{~m} \cdot \mathrm{s}^{-1}$; su región oceánica presentó vientos más intensos en toda la CPC. Lo anterior indica que se siguieron incrementando los vientos Alisios del sur, y los Alisios del Norte mantuvieron su incidencia aunque no muy significativa, siendo su componente zonal mayor que el meridional.

Teniendo en cuenta el campo de vientos para agosto mediante información in situ (Fig. 6) se registraron vientos predominantes del Oeste en la mayoría de estaciones de la región central y Norte de la CPC. La región Sur de la CPC registró vientos del suroeste que se ajustaron mejor a la dinámica vectorial de los vientos para esta época del año, a diferencia de las otras regiones de estudio sobre la CPC.

\section{Septiembre}

El comportamiento de los vectores de viento para septiembre presentó la mayor influencia de los vientos Alisios del sur, sobre la CPC y sobre el golfo de Panamá. Es posible visualizar en el campo de vientos de este mes el debilitamiento total del chorro de Panamá (Fig. 5)

La ZCN registró vientos con intensidades entre los 6 a $6.5 \mathrm{~m} \cdot \mathrm{s}^{-1}$, y en su sector oceánico presentó una gradual disminución llegando hasta los $5 \mathrm{~m} \cdot \mathrm{s}^{-1}$. En la ZCC se registraron velocidades entre los 6 y $9 \mathrm{~m} . \mathrm{s}^{-1}$, su región oceánica presentó intensidades un poco más bajas con relación a las observadas cerca al litoral. La ZCS mostró las mayores intensidades, con valores entre los 7 y $9 \mathrm{~m}^{-s^{-1}}$, las cuales disminuyen a medida que se alejan de la costa. La dirección predominante para estos vectores es suroeste.

Se observó en los vectores de viento superficiales obtenidos a partir de información in situ (Fig. 6) un predominio de vientos del suroeste en toda la CPC. En el litoral Pacífico colombiano, específicamente en la ZCC, se registraron vientos predominantes del oeste, los cuales transportan humedad hacia la costa, influyendo directamente sobre los índices de precipitación producto de la condensación resultante.

Finalmente, para este mes el chorro de Panamá se debilitó por completo, por lo que la CPC y el golfo de Panamá registraron el predominio de vientos Alisios del sur. Por otro lado, el chorro del Chocó manifestó su mayor actividad evidenciada con vientos Alisios del suroeste con velocidades entre los 6 a $9 \mathrm{~m} \cdot \mathrm{s}^{-1}$, sobre la CPC.

\section{Octubre}

El comportamiento general del campo de viento para este mes mantuvo un predominio de los vientos Alisios del Sur sobre la CPC y el golfo de Panamá (Fig. 5). El comportamiento del viento fue similar al de septiembre. Las intensidades de los vientos Alisios del Sur sobre la CPC y el golfo de Panamá comenzaron a disminuir a partir de este mes, dándose inicio a la reactivación del chorro de Panamá.

La información in situ de octubre (Fig. 6) registró predominio de los vientos Alisios del Sur en la CPC, aunque se presentaron registros de vectores predominantes del Oeste en la región oceánica y vectores de viento del este en áreas cercanas a la costa. 

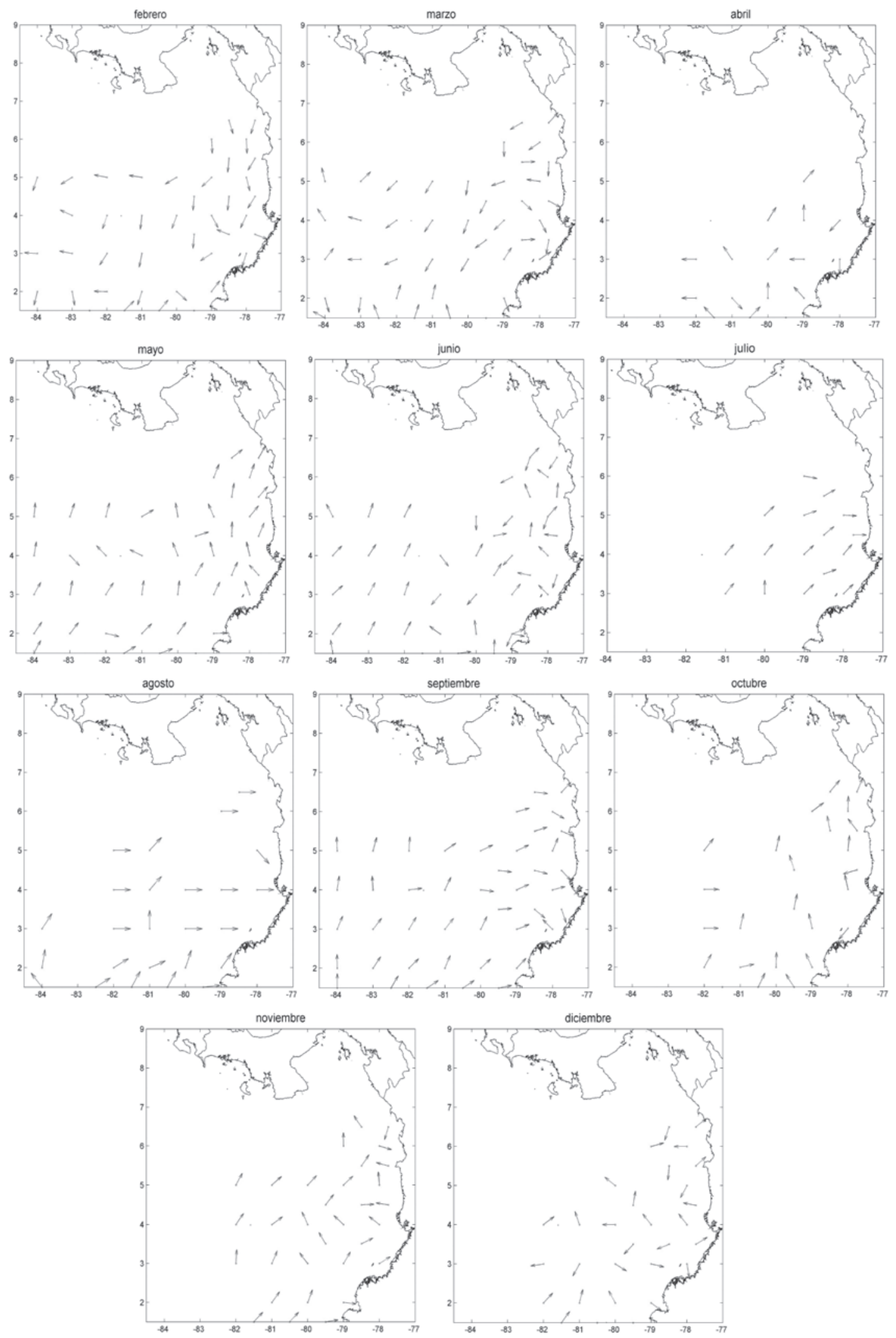

Figura 6. Serie de tiempo de datos in situ CCCP para el período de 53 meses (1972 - 2007) del campo de vientos promedio mensual en la región de la CPC. 


\section{Noviembre}

Para noviembre el campo de vientos presentó la reactivación del chorro de Panamá (Fig. 5). Vientos del noroeste y oeste, con velocidades entre los 4.5 y $5.5 \mathrm{~m} . \mathrm{s}^{-1}$, incursionaron al golfo Panameño. Los vientos Alisios del Norte se reactivaron, por lo que los Alisios del Sur disminuyeron su influencia sobre la CPC y el golfo.

En el caso de la ZCN se presentó un debilitamiento en las intensidades del viento en su región costera; asimismo, se registraron variaciones en la dirección del viento, las cuales pasan de incursionar del suroeste a predominar del oeste. La ZCC presentó, al igual que la ZCN, una disminución en las intensidades del viento, con valores entre los 8 y $6 \mathrm{~m} . \mathrm{s}^{-1}$; los vectores predominantes siguen siendo del sur-oeste, indicando variaciones no muy significativas. Finalmente, en la ZCS se presentó una dinámica similar a la de la zona centro y Norte del litoral Pacífico colombiano.

Los vectores de vientos obtenidos para noviembre a partir de información in situ (Fig. 6) registraron el predominio de vientos del suroeste, similar al mes anterior, disminuyendo gradualmente sus intensidades.

\section{Diciembre}

El comportamiento de los vectores de viento diciembre presentó la continua reactivación del chorro de Panamá y la disminución de advección de humedad por los vientos del chorro del Chocó (Fig. 5)

La ZCN presentó un debilitamiento notorio, con velocidades entre los 2 y $4 \mathrm{~m} \cdot \mathrm{s}^{-1}$ y vientos predominantes del este, noroeste y suroeste cercanos a la costa, por lo que la ZCIT inició su reaparición sobre la CPC, actuando directamente sobre esta región del litoral colombiano. La ZCC presentó vientos con intensidades entre los 4.5 y $5.5 \mathrm{~m} . \mathrm{s}^{-1}$, procedentes del suroeste. Su sector oceánico registró vientos predominantes del oeste, con velocidades similares a las presentadas en el litoral. Finalmente, para este mes empezó a definirse claramente la ZCIT, la cual reaparece con intensidades entre los $1 \mathrm{a} 2 \mathrm{~m} . \mathrm{s}^{-1}$ cercanos a la costa y 4 a $5 \mathrm{~m} \cdot \mathrm{s}^{-1}$ en la región oceánica.

El comportamiento en los vectores de viento a partir de datos in situ para este mes (Fig. 6) exhibió una irregularidad en dirección sobre la
CPC, debido principalmente a la reactivación del chorro de Panamá (Fig. 5) que alteró el flujo regular de los vectores de viento sobre la cuenca colombiana.

\section{DISCUSIÓN}

\section{Magnitudes anuales, semestrales y su relación con el chorro del Chocó}

Los vientos Alisios del sur, que fluyen paralelos a la costa este del Pacífico Sur, sufren una recurvatura al cruzar la línea Ecuatorial (Poveda y Mesa 1997, 1999; Poveda 2004; Poveda et ál ., 2006), los cuales disminuyen su componente meridional, incrementando su componente zonal hacia el este. Este proceso físico de recurvatura se explica en el trabajo realizado por Hanstenrath en 1991, mediante la ecuación para la determinación de la dinámica de los flujos trans-ecuatoriales.

$\mathrm{El}$ anterior comportamiento se registra en las imágenes de magnitud anual y semestral del componente zonal, el cual predomina sobre toda la CPC (Fig. 7). Con relación al componente meridional, en su magnitud anual, los vientos predominaron del sur, en toda la CPC, hasta llegar al golfo de Panamá. En la magnitud semestral, en el primer semestre se registra la influencia del chorro de Panamá, sobre la CPC, principalmente sobre la región oceánica Norte de la misma (Fig. 7b)

La persistencia zonal oeste sobre la CPC genera fenómenos océano-atmosféricos, tales como el chorro del Chocó. Este chorro de vientos superficiales es generado por el constante flujo de vientos Alisios del suroeste, en dirección a la costa Pacífica colombiana. El chorro del Chocó transporta una gran cantidad de humedad al interior de Colombia, generando procesos convectivos sobre la región Pacífica (Poveda 2004) y otras regiones cerca al área de influencia, como son Los Andes y la Orinoquía.

La corriente del chorro del Chocó obtiene su energía del gradiente de temperaturas superficiales entre la zona de temperaturas menos cálidas de la llamada lengua fría, provenientes de las aguas del Pacífico en Ecuador y Perú, y temperaturas más cálidas provenientes de las costas colombianas. Este gradiente de temperaturas se encuentra asociado a las presiones atmosféricas superficiales, las cuales inducen a la recurvatura presentada en los vientos 
Alisios transecuatoriales, ingresando por el Oeste de Colombia (Poveda, 2004)

Las magnitudes semestrales registran vientos del Norte durante el primer semestre, para posteriormente predominar los del Sur en el segundo; además, el flujo zonal Oeste participó como el principal componente del chorro del Chocó, el cual disminuye en el primer semestre, para incrementarse nuevamente durante el segundo (Fig. 7). Las magnitudes semestrales obtenidas se relacionan a los estudios realizados por Poveda (2004), donde se hace mención al debilitamiento del chorro del Chocó, presentando además sus mayores intensidades entre septiembre y noviembre. El anterior debilitamiento es producto principalmente de la disminución del gradiente entre las TSM de la costa Perú-Ecuador y Colombia sobre el Pacífico, así como por la influencia del chorro de Panamá entre diciembre y febrero (Fig. $5)$, que ejerce una disminución en los componentes meridional Sur y zonal Oeste del viento sobre la CPC (Fig. 7b)

Con relación a la información in situ, ésta se asemeja con la obtenida por el dispersómetro (Fig. 6). En febrero y marzo los vectores de viento fueron predominantes del norte, con velocidades promedio entre 4.5 a $6 \mathrm{~m} . \mathrm{s}^{-1}$ sobre la CPC (figuras 6 , 8 y 12a), debilitando el chorro de Chocó. A partir de mayo (figuras 6 y 12a) empiezaron a registrarse vientos del Sur sobre la CPC. Cabe notar además que abril (Fig. 6) se puede considerar como un mes de transición de vientos predominantes del Norte a vientos procedentes del Sur sobre la CPC.

Entre agosto y noviembre se presentó la mayor influencia de vientos del suroeste sobre la costa Pacífica colombiana (figuras 5 y 6 ), principalmente sobre las regiones de la ZCS y ZCC, con velocidades promedio de 6 a $9 \mathrm{~m} \cdot \mathrm{s}^{-1}$, de agosto a octubre, disminuyendo ligeramente para noviembre.

La anterior dinámica del campo de vientos permite regular los procesos convectivos de mesoescala sobre la región, en directa cooperación dinámica y termodinámica con el chorro del Chocó; por lo que es de gran importancia conocer los comportamientos anual y semestral del componente zonal y meridional en la CPC, ya que estos generan procesos océano-atmosféricos que regulan los parámetros hidrometeorológicos de la costa Pacífica colombiana y el interior del país.
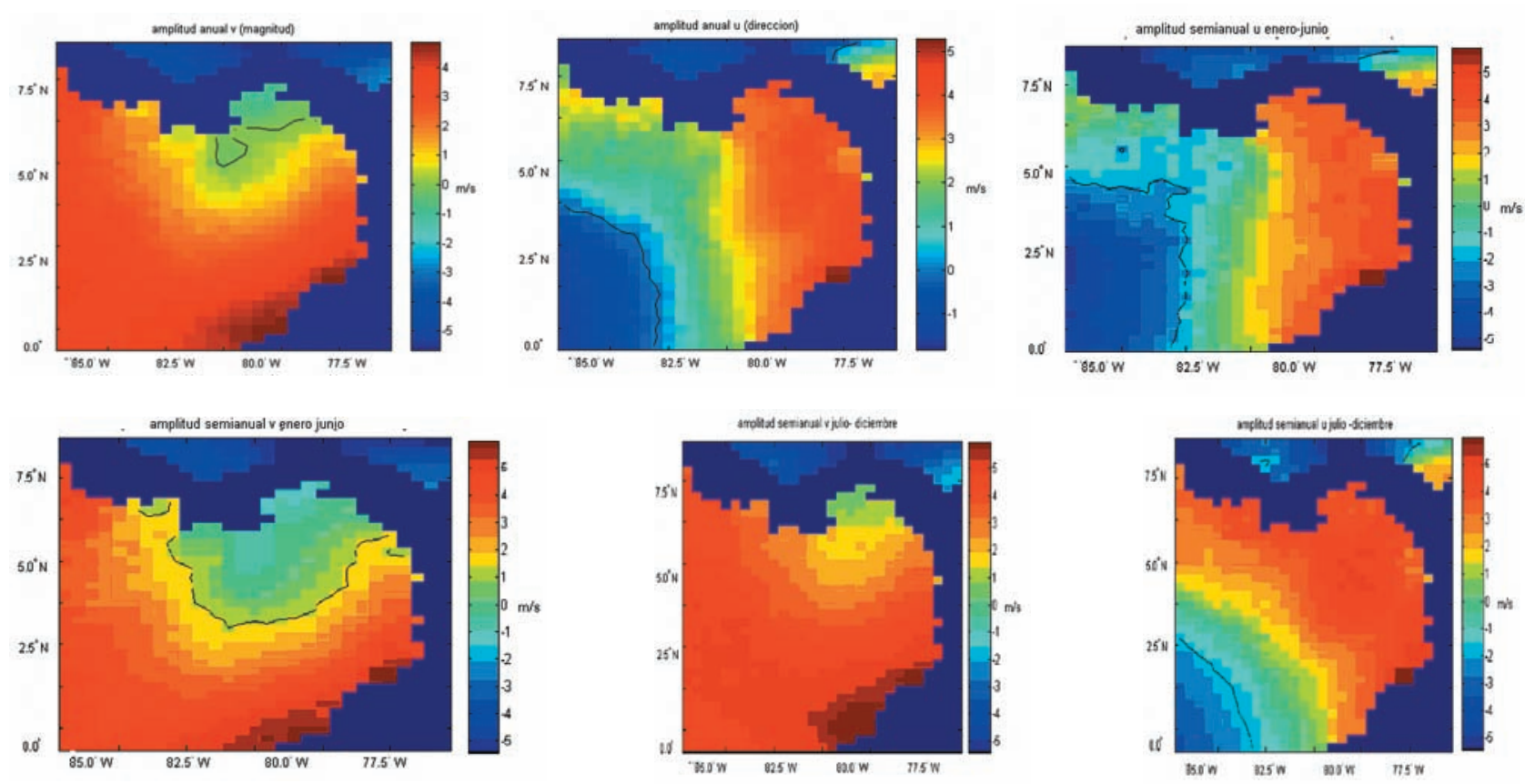

Figura 7. (a) Magnitud anual meridional $v$ y zonal u obtenidos del sensor QSCAT para el período de 65 meses (Jul. 1999 a Dic. 2005). (b) Magnitud semestral (Ene. - Jun.) zonal u y meridional v 1999-2005 QSCAT. (c) Magnitud semestral (Jul. - Dic.) zonal u y meridional v para el período 1999 - 2005 provenientes del sensor QSCAT. 
Relación entre el campo de vientos y TSM durante marzo de 2006

En marzo de 2006 la DIMAR realizó el crucero oceanográfico número 42 en la CPC. Los resultados

A

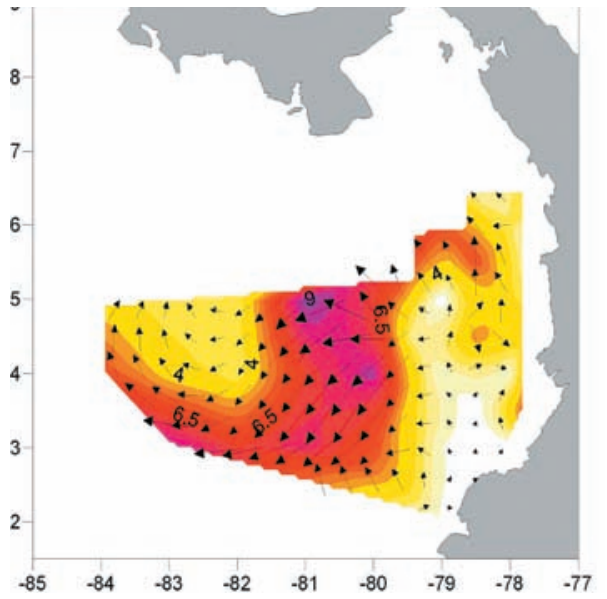

obtenidos indicaron que el comportamiento del campo de vientos es similar al de la climatología in situ.

En el centro de la CPC se ve la influencia directa del chorro de Panamá sobre la cuenca colombiana.

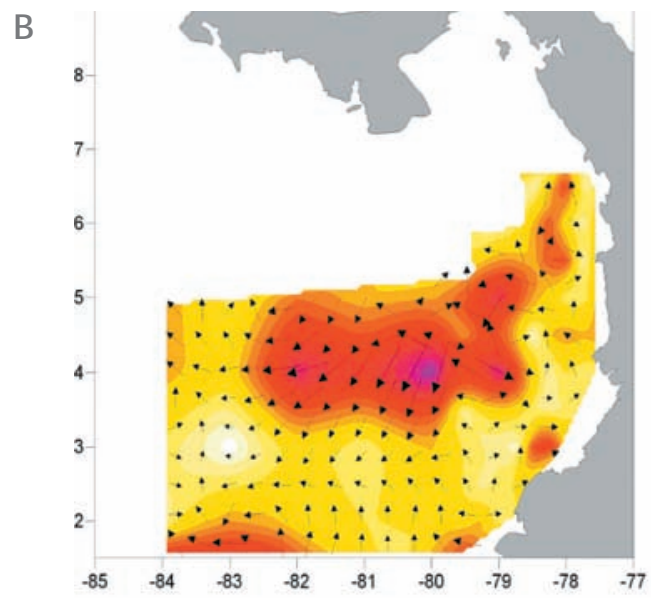

Figura 8. Campo de vientos de marzo en la CPC (a), climatología in situ (b) 2006.

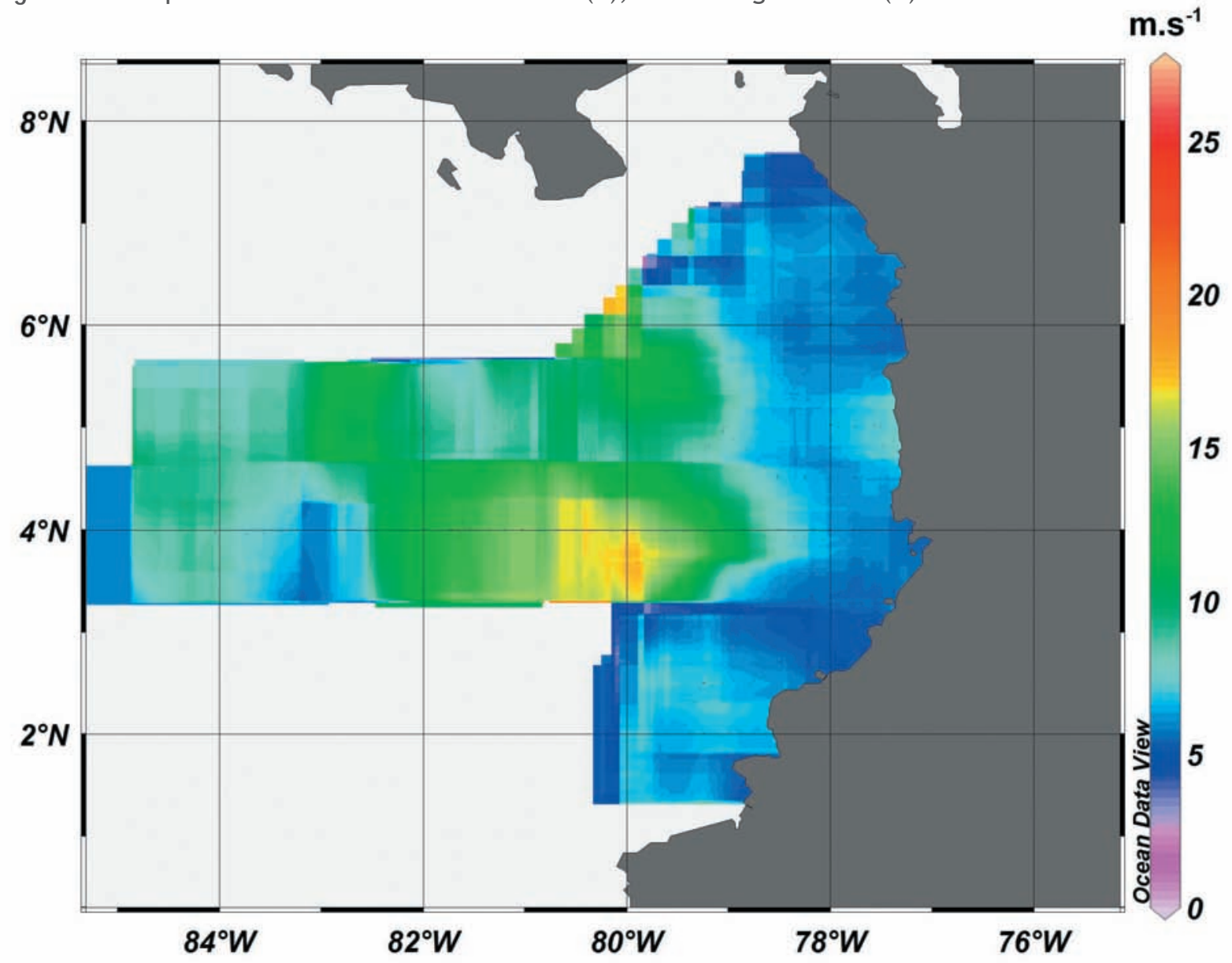

Figura 9. Registro horario de la velocidad del viento durante el crucero oceanográfico de marzo de 2006 en la CPC. 


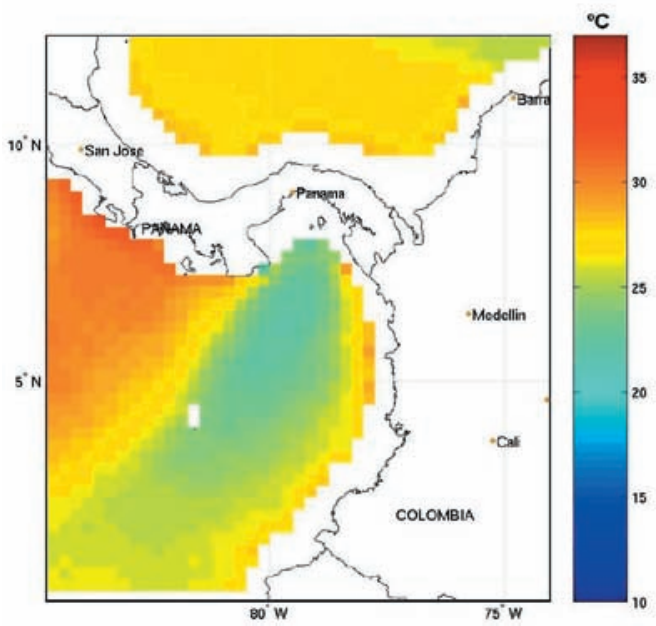

Figura 10. TSM promedio mensual de marzo de 2006 TMI (Tomado de: Bastidas, 2006)

En las imágenes de datos in situ por estación (Fig. 8), tanto en la climatología como en marzo de 2006 se registran vientos entre los 5 y $9 \mathrm{~m} \cdot \mathrm{s}^{-1} \mathrm{en}$ el centro de la CPC. La imagen de magnitud obtenida a partir información in situ, registrada cada hora durante el crucero oceanográfico realizado en marzo de 2006 (Fig. 9) presenta también las mayores magnitudes de vientos en el centro de la CPC con velocidades entre los 8 y $16 \mathrm{~m} \cdot \mathrm{s}^{-1}$.

El comportamiento vectorial de la información in situ (Fig. 8) indica que los vientos predominantes en el centro de la CPC son del noreste. Se puede notar además que los vientos Alisios del noreste convergen con los vientos Alisios del Sur entre las latitudes $2^{\circ} 30^{\prime}$ y $3^{\circ} \mathrm{N}$, tanto en la imagen de la climatología como en la del año 2006. Se observó además que entre los meridianos $79^{\circ}$ y $79^{\circ} 30^{\prime} \mathrm{W}$ se registra un comportamiento divergente de los vientos Alisios del sur, los cuales tienden hacia el Oeste y al este al mismo tiempo, registrando magnitudes entre los 2 y $4 \mathrm{~m} . \mathrm{s}^{-1}$ (Fig. 8)

Con relación al comportamiento de los vectores de viento en las regiones cercanas al litoral Pacífico colombiano, estos presentan irregularidad en su dirección, aunque los más cercanos a la costa predominan del Oeste y suroeste. Las magnitudes registradas cerca del litoral fueron bajas, presentando velocidades entre 1 y $5 \mathrm{~m} . \mathrm{s}^{-1}$.

La TSM de marzo de 2006 (Fig. 10) registró un núcleo de aguas frías con valores entre los 22 y $24{ }^{\circ} \mathrm{C}$ en el centro de la CPC y en gran parte del golfo de Panamá. Este comportamiento evidencia la incidencia del chorro de Panamá (Fig. 5) sobre las áreas de estudio, por lo que esta influencia de vientos sobre las aguas del golfo Panameño y la CPC permite el ascenso de aguas profundas, las cuales disminuyen la TSM en la región (Kwiecinski et ál., 1975; Legekis, 1985). Esta disminución en la TSM influye directamente sobre la energía del chorro del Chocó, teniendo en cuenta que el gradiente de temperatura entre las aguas del Pacífico ubicadas cerca de Ecuador, Perú y las aguas cercanas a la costa de Colombia es reducido, principalmente el enfriamiento de las aguas superficiales en la CPC durante los primeros meses del año.

\section{Análisis de la dirección promedio, persistencia y probabilidad de los vectores de viento in situ}

El comportamiento de la serie de tiempo de la Figura 11(a) registró en febrero una dirección promedio de $27^{\circ}$ en el sentido nor-noreste. A partir de febrero y hasta mayo los vectores efectúan un giro gradual en sentido a las manecillas del reloj de $177^{\circ}$, registrándose en mayo un vector promedio de $204^{\circ}$ en sentido sur-suroeste. Partiendo de mayo hasta llegar al registro de $246^{\circ}$ en agosto, se mantiene el predominio de vientos del suroeste. Finalmente, iniciando desde agosto hasta llegar al último registro de $156^{\circ}$ en sentido sur-sureste de diciembre, se mantiene el predominio de vientos Alisios del Sur en la CPC.

Con respecto a la persistencia del viento (Fig. 11b) los registros más bajos se presentaron durante marzo, abril y diciembre, debido a la gran variabilidad vectorial manifestada durante estos meses. La estabilidad en el comportamiento de la serie de tiempo de la persistencia del viento inicia en julio con un registro de 91.35 , y se extiende hasta noviembre con un registro de 62.80. La anterior estabilidad se debe principalmente al predominio de vientos Alisios del Sur (figuras 5 y 6), los cuales mantienen una regularidad en los componentes meridionales y zonales sobre la región.

La probabilidad presentada en la serie (Fig. 11c) indica que para el período de mayo a noviembre la probabilidad de las componentes zonales y meridionales es alta. Lo anterior se puede explicar a través de la influencia del chorro de Panamá, teniendo en cuenta que su mayor incidencia sobre la CPC se registra durante el período comprendido 
a

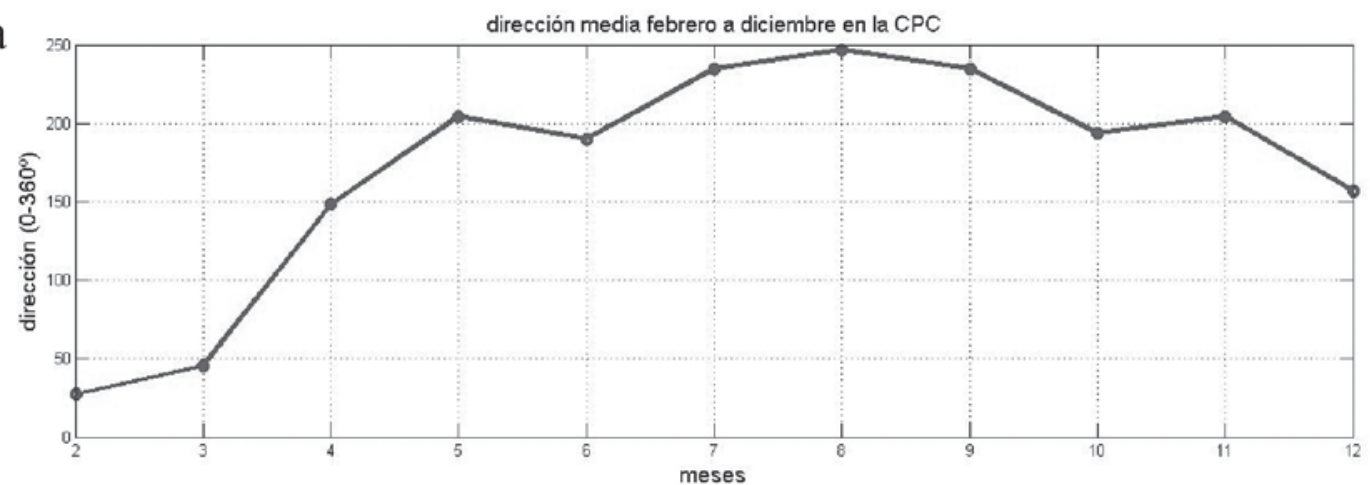

$\mathrm{b}$
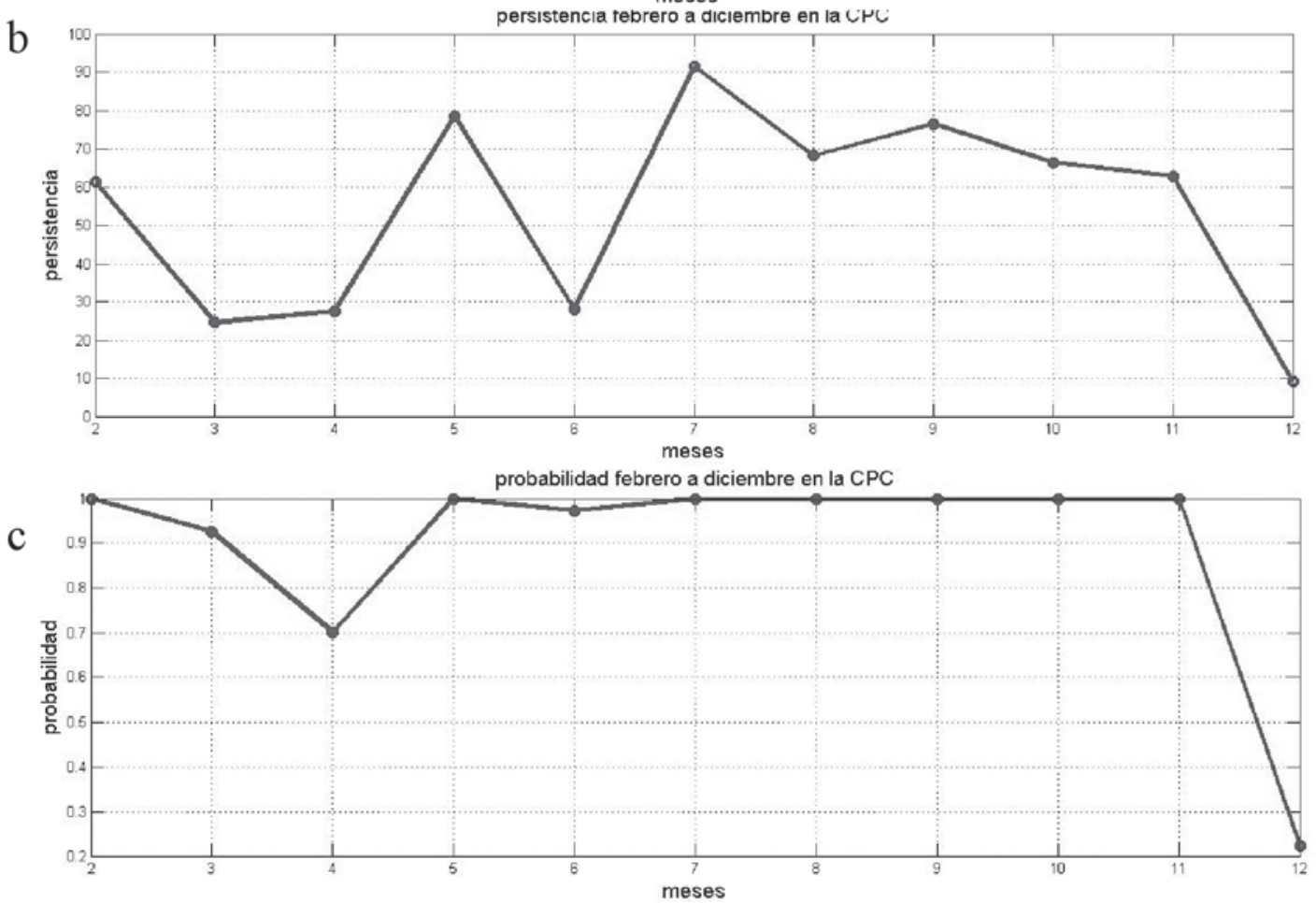

Figura 11. (a) Dirección promedio del viento horizontal. (b) Persistencia. (c) Probabilidad del viento. Datos in situ para el período febrero-diciembre.

entre diciembre y abril (figuras 5 y 6), manteniéndose ausente entre mayo y noviembre. El anterior comportamiento indica que este chorro de viento altera el flujo regular de los vectores de viento superficiales del suroeste durante los primeros meses del año.

\section{CONCLUSIONES}

- Las correlaciones efectuadas entre la información de la dirección de viento obtenida durante los cruceros oceanográficos (CCCP) con respecto al sensor remoto (QSCAT) indican una alta correlación en gran parte del año, exceptuando los meses de abril, mayo, noviembre y diciembre, que indicaron baja correlación y tampoco alcanzaron a rechazar la hipótesis de independencia en los datos.

- La climatología obtenida indica que el golfo de Panamá presenta un comportamiento estacional semestral. Durante el primer semestre predominan los vientos Alisios del noroeste, con velocidades entre

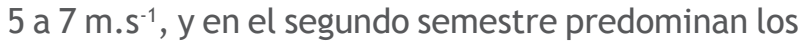
vientos Alisios del suroeste con intensidades entre 4 y $5 \mathrm{~m} \cdot \mathrm{s}^{-1}$.

- Las magnitudes semestrales del campo de 
vientos de la CPC indican en el primer semestre la influencia del chorro de viento de Panamá entre enero y abril, ingresando a la CPC con vientos entre 5 a $9 \mathrm{~m} . \mathrm{s}^{-1}$ predominantes del noroeste, Norte y noreste. Durante el primer semestre el chorro de viento de Panamá regula la manifestación de la ZCIT en la CPC, mostrando el desplazamiento de un cinturón de bajas velocidades con valores entre 0 a $4 \mathrm{~m} \cdot \mathrm{s}^{-1}$.

- Con relación al campo de vientos de la CPC durante el segundo semestre se registra el predominio de vientos del suroeste con velocidades entre 5 a $9 \mathrm{~m} . \mathrm{s}^{-1}$, a excepción de la ZCN en diciembre, registrándose vientos variables del oeste, noroeste y suroeste con velocidades entre $2 \mathrm{a} 4 \mathrm{~m} . \mathrm{s}^{-1}$.

- Las magnitudes anuales indicaron un predominio de vientos del suroeste en la mayoría de las regiones de la CPC, con velocidades entre 1 y $5 \mathrm{~m} \cdot \mathrm{s}^{-1}$, encontrándose los mayores registros de velocidad en las zonas Sur y centro de la misma.

- Durante el primer trimestre del año las aguas superficiales en la CPC se enfrían a causa de la activa y continua influencia del chorro de viento de Panamá, disminuyendo el gradiente de temperaturas existente entre las aguas de la CPC y las aguas de Perú y Ecuador. Esta disminución en la TSM, evidenciada en la relación efectuada entre el campo de vientos y la TSM durante marzo del 2006, genera una reducción en la energía del chorro del Chocó. Durante el segundo semestre el chorro de Panamá se debilita, permitiendo el incremento de los vientos Alisios del Sur y el aumento de las TSM en la CPC, dando como resultado la intensificación del gradiente de temperaturas en la región y por ende la intensificación e incursión del chorro el Chocó a la CPC y al litoral Pacífico colombiano.

- La serie de tiempo mensual de dirección promedio del viento in situ exhibe una irregularidad vectorial en los primeros meses del año, dando como resultado a una baja persistencia y probabilidad de los mismos. Esta irregularidad se justifica por la influencia que ejerce el chorro de Panamá sobre la CPC a través de la actividad convectiva de la ZCIT.

- Durante el período mayo-noviembre predominan los vientos del Sur sobre la CPC, registrándose regularidad en la persistencia del viento de julio a noviembre, comportamiento que es consistente con la probabilidad misma de los vectores de viento. Esta regularidad es debida al constante flujo de los vientos Alisios del suroeste, los cuales durante el segundo semestre mantienen un comportamiento vectorial con pocas variaciones en velocidad y dirección (chorro del Chocó); caso contrario a diciembre, mes en el que se inicia la reactivación del chorro de Panamá hasta abril. Durante la reactivación del chorro de Panamá la persistencia disminuye de 62.8 , en noviembre, hasta 10 , en diciembre, y la probabilidad del viento de 0.999 hasta 0.23 para el mismo período.

- Teniendo en cuenta los resultados de dirección media, el giro gradual de $117^{\circ}$ obtenido en mayo permite identificar un cambio en la componente meridional, predominando del Norte de diciembre a febrero sobre la CPC; varía su predominio con vientos del Sur de mayo a noviembre sobre la región. Estos cambios en la dirección media indican la manifestación del chorro de Panamá y del chorro del Chocó.

- Se recomienda incrementar los muestreos en los meses con bajo cubrimiento de estaciones de muestreo, como también empezar por realizar muestreos en enero, sobre el cual no hay registro alguno sobre la CPC. Igualmente, es importante incrementar el uso de las herramientas tecnológicas en la medición del viento para obtener una mayor frecuencia y resolución espacial en las zonas costeras del Pacífico colombiano.

- Conviene iniciar mediciones y estudios del campo de vientos vertical en la región, ya que no se posee información sobre el comportamiento del viento en el eje vertical. Este tipo de mediciones permite explicar la generación de sistemas convectivos de mesoescala y su interacción con la dinámica del chorro del Chocó y el chorro de Panamá en la región.

\section{AGRADECIMIENTOS}

Este trabajo fue financiado por el proyecto Ampliación de Conceptos Oceanográficos de la Cuenca Pacífica Colombiana de la DIMAR-CCCP.

Los autores expresan sinceros agradecimientos a los suboficiales de la Armada Nacional Federico Castillo y Oscar Fajardo, a la ingeniera física Martha Lucero Bastidas Salamanca, al biólogo marino Julián Uribe y la señora Lida Arroyo por el apoyo técnico y profesional brindado en el desarrollo del presente estudio. 


\section{LITERATURA CITADAA}

Bastidas, M. L. 2006. Seguimiento satelital de los parámetros TSM y clorofila_a superficial durante marzo y septiembre del 2006 en la CPC y sus áreas insulares. Centro Control Contaminación del Pacífico. Informe técnico. San Andrés de Tumaco: CCCP, Colombia.

Centro Control Contaminación del Pacífico. 2002. Compilación Oceanográfica de la Cuenca Pacífica Colombiana. (Ed.) DIMAR. San Andrés de Tumaco: CCCP, Serie Publicaciones Especiales Vol. 1, p. 16.

Chelton, D., H. Freilich y K. Esbensen. 2000a. Satellite Observations of the Wind Jets Off the Pacific Coast of Central America. Part II: Relationships and Dynamical Considerations. Mon. Wea. Rev., (128): 2019-2043.

Chelton, D., H. Freilich y K. Esbensen. 2000b. Satellite Observations of the Wind Jets Off the Pacific Coast of Central America. Part I: Case Studies and Statistical Characteristics. Mon. Wea. Rev., (128): 1993-2007.

Eslava, J. 1994. Climatología del Pacífico colombiano. Acad. Colomb. Cienc. Geof., No 1, Bogotá, 79 pp.

Forsbergh, E. D. 1969. On the Climatology, Oceanography and Fisheries of the Panama Bight. Bull. Inter.-Am. Trop. Tuna Comm. 14 (2): 49-385.

Garcés-Vargas, J , R. Abarca del Río y W. Schneider. 2004. [en línea].Climatology and interannual variability of Intertropical Convergence Zone in the eastern Pacific. Gayana (Concepc) Vol. 68, No.2, supl. pp.215-217 [Consulta: 15 de junio 2006], . Disponible en: <http://www.scielo.cl/scielo. php?script $=$ sci_arttext\&pid $=$ S0717 -6538200400 0200039\&lng=es\&nrm=iso>

Hellerman, S. y M. Rosenstein. 1983. Normal Monthly Windstress over the World Ocean with Errors Estimates. Journal Physical Oceanographic, (13): 1093-1104.

J ones, T. A. 2006. MATLAB functions to analyze directional (azimuthal) data -II: Correlation. Computer \& Geosciences, (32): 176-183.

Kwiecinski, B., A. J aen y A. Moschett. 1975. [en línea]. Afloramiento en el Golfo de Panamá durante la temporada de 1973. An. Centro Cienc. del Mar y Limnol. Univ. Nal. Auton. México 2 (1): 73-80, [Consulta: 14 de junio de 2006]. Disponible en: http://llyncis.dgsca.unam.mx:8080/examples/ servlet/ciencias.FichasCiencias2?xml=archivo12. $\mathrm{xml}$.

Ledoux, H. y C. Gold. 2004. An efficient natural neighbour interpolation algorithm for geoscientific modelling. En: Peter, F., Fisher (ed.), Developments in Spatial Data Handling-11th International Symposium on Spatial Data Handling, pp. 97-108.

Legekis, R. 1985. Upwelling off the Gulfs of Panam and Papagayo in the Tropical Pacífic During March. Journal Geophysical Reseach, 93. No.C12, 1988, pp. 15,485-15,489.

Málikov, I. 1998. Determinación de los cambios entre anuales del campo de presión atmosférica en el Pacífico colombiano. Informe técnico. Centro Control Contaminación del Pacífico. San Andrés de Tumaco: CCCP, Colombia.

Mardia, K. V. y P. E. J upp. 2000. Directional statistics. Wiley, Chichester, UK 429 pp.

McCreary, J. P., H. S. Lee y D. B. Enfield. 1989. The Response of the Coastal Ocean to Strong Offshore Winds: With Application to Circulations in the Gulfs of Tehuantepec and Papagayo. J ournal Marine Research, (47): 81-109.

Middleton, G. 2000. Data Analysis in the earth sciences using Matlab. Prentice-Hall, Inc. Upper Saddle River, New Jersey 07458. USA.

Philander, S. G. 1990. El Niño, La Niña, and the Southern Oscillation. Academic Press, San Diego, $293 \mathrm{pp}$.

Poveda, G. 2004. [en línea]. La Hidroclimatología de Colombia: una síntesis desde la escala interdecadal hasta la escala diurna. Revista Colombiana de las Ciencias Exactas, Físicas y Naturales. Índice 
del Vol. XXVIII (No. 106, 107). [Consulta: 13 de junio de 2006]. Disponible en:

http: / / www.accefyn.org.co/PubliAcad/ Periodicas/indice(28).html.

Poveda G. y O. J . Mesa. 1997. Feedbacks between hydrological processes in tropical South America and large scale oceanic atmospheric phenomena. Journal of Climate, Vol. 10, No. 10, 2690-2702.

Poveda, G. y 0. J. Mesa. 1999. La Corriente de chorro Superficial del Oeste (“del CHOCÓ”) y otras dos corrrientes de chorro atmosféricas sobre Colombia: Climatología y Variabilidad durante las fases del ENSO. Revista de la Academia Colombiana de Ciencias Exactas Físicas y Naturales, Vol. 23, No. 89, 517-528.

Poveda, G. y 0. J. Mesa. 2000. On the existence of Lloró (the rainiest locality on Earth): Enhanced ocean-atmosphere-land interaction by a low-level jet. Geophysical Research Letters, Vol. 27, No. 11, 1675-1678.

Robinson, I. 1994. Satellite oceanography: an introduction for oceanographers and remotesensing scientists. Chichester, Eng., E. Horwood; Chichester, Eng., New York, Wiley-Praxis, 455 pp.

Rodríguez-Rubio, E. y J . Stuardo. 2002. Variability of photosynthetic pigments in the Colombian Pacific Ocean and its relationship with the wind field using ADEOS-I data. Proc. Indian Acad. Sci. (Earth Planet. Sci.), 111, No. 3, pp. 227-236.

Rodríguez-Rubio, E., W. Schneider y R. Abarca del Río. 2003. On the seasonal circulation within the Panama Bight derived from satellite observations of wind, altimetry and sea surface temperature, Geophys. Res. Lett., 30 (7), 1410, doi: 10.1029/2002GL016794.

Smayda, T. J. 1966. A quantitative analysis of the phytoplankton of the Gulf of Panama.
III. En: General, Ecological conditions and the phytoplankton dynamics at $08^{\circ} 45^{\prime} \mathrm{N}, 79^{\circ} 23^{\prime} \mathrm{W}$ from November 1954 to May 1957 (with Spanish summary). Bull. Inter. Amer. Trop. Tuna. Comm. (11): 353-612.

Tokioka, T. 1983. [en línea]. Influence of the ocean on the atmospheric global circulations and short-range climatic fluctuations. G. D. Sharp and J. Csirke (eds.). Proceeding of the expert consultation to examine change in abundance and species of neritic fish resources. San José de Costa Rica. FAO Fish. Rep., 291(3): 557-585. [Consulta: 14 de junio de 2006]. Disponible en: http://www.fao. org/DOCREP/003/X 6851B/X6851B01.htm\#ch1.1.1

Topliss, B. J. y H. Guymer. 1995. Marine Winds from scatterometers. Pp. 205-221. In: M. Ikeda and F. W. Dobson [eds.], Oceanographic applications of remote sensing. CRC Press.

Umatani, S. y T. Yamagata. 1991. Response of the Eastern Tropical Pacific to Meridional Migration of the Itcz: The Generation of the Costa Rica Dome. Journal Physical Oceanographic (21): 346-363.

Villegas, N. 2003. Estabilidad de las aguas del Pacífico Colombiano. En: Boletín Científico CCCP, (10): 1-11.

Waliser, D. E. y R. C. J . Somerville. 1994. Preferred Latitudes of the Intertropical Convergence Zone. En: Journal Atmospheric Science, 51 (12): 16191639.

Wang, C. y S. Lee. 2007. Atlantic warm pool, Caribbean low-level jet, and their potential impact on Atlantic hurricanes. En: Geophysical Research Letters, Vol. 34, L02703, doi:10.1029/ 2006GL028579. 